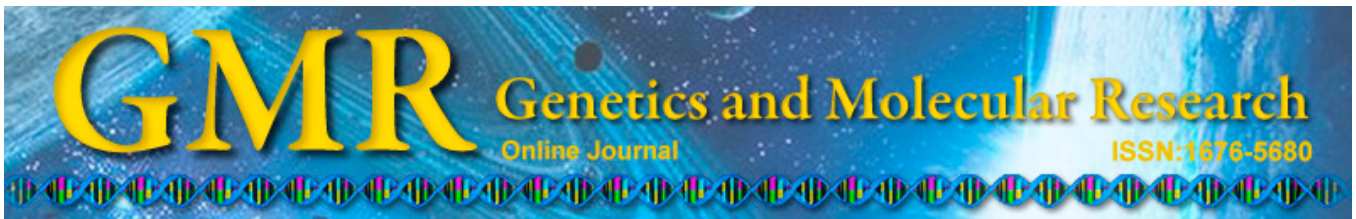

\title{
VDAC2 involvement in the stress response pathway in Arabidopsis thaliana
}

\author{
Z. Liu ${ }^{1,2}$, Q.H. Luo ${ }^{3}$, G.Q. Wen ${ }^{4}$, J.M. Wang ${ }^{1}$, X.F. Li ${ }^{1}$ and Y. Yang ${ }^{1,2}$ \\ ${ }^{1}$ Key Laboratory of Bio-Resources and Eco-Environment, \\ Ministry of Education, College of Life Sciences, Sichuan University, \\ Chengdu, Sichuan, China \\ ${ }^{2}$ State Key Laboratory of Hydraulics and Mountain River Engineering, \\ Sichuan University, Chengdu, Sichuan, China \\ ${ }^{3}$ Longquanyi District Bureau of Agriculture Development, Chengdu, Sichuan, \\ China \\ ${ }^{4}$ College of Life Science, China West Normal University, Nanchong, Sichuan, \\ China \\ Corresponding author: Y. Yang \\ E-mail: yangyi528@scu.edu.cn
}

Genet. Mol. Res. 14 (4): 15511-15519 (2015)

Received August 13, 2015

Accepted October 19, 2015

Published December 1, 2015

DOI http://dx.doi.org/10.4238/2015.December.1.1

\begin{abstract}
The voltage-dependent anion channel (VDAC) is the major transport protein in the outer membrane of mitochondria, and is involved in the formation of a permeable transition pore and metabolite transport. In this study, we explored the role of Arabidopsis thaliana VDAC2 (AtVDAC2) in the signal transduction pathway in Arabidopsis under salt stress. We investigated the germination rates of AtVDAC2 transgenic lines under salicylic acid (SA) treatment, and found that AtVDAC2 can affect the sensitivity of Arabidopsis to SA. Furthermore, the stomatal apertures of AtVDAC2 transgenic Arabidopsis were calculated. Results showed that the over-expression lines showed the obvious stomatal closure, while in the antisense lines, no obvious changes in stomatal apertures were found. In addition, we also detected the expression levels of salt stress and SA response-associated genes
\end{abstract}


in transgenic plants and found that AtVDAC2 affected the expression of these genes. Our study investigated the role of AtVDAC2 in SA and salt stress response in Arabidopsis; our observations provide some helpful information for better understanding the direct and downstream functions of AtVDAC.

Key words: Arabidopsis; VDAC; Salicylic acid; Salt stress

\section{INTRODUCTION}

Mitochondria are among the most important cellular organelles, as they regulate cellular respiration, apoptosis, and other life processes. Mitochondrial proteins are primarily located at the inner mitochondrial membrane, which regulates the metabolic pathways and biological processes of the mitochondria (Douce, 1985; Douce and Neuburger, 1989; Mackenzie and McIntosh, 1999; Moller, 2001; Dudkina et al., 2006). The mitochondrial outer membrane contains only a small quantity of proteins. Among them, the voltage-dependent anion channel (VDAC) protein is the most crucial protein in plants. VDAC regulates a variety of physiological and pathological processes by controlling mitochondrial and cytoplasmic transport of substances (Kroemer et al., 2007). Programmed cell death (PCD) has been found to occur under environmental changes or at specific developmental stages in plants. Many studies have found that PCD in plants is similar to cellular apoptosis in mammals, and that VDAC genes play a vital role in regulating the PCD process (Pennell and Lamb, 1997; Hofius et al., 2007; Bonneau et al., 2008; Love et al., 2008; Reape and McCabe, 2008; Kusano et al., 2009).

Salt is the major factor limiting plant growth and productivity. To adjust to salt stress, plant cells activate a series of regulatory pathways. First, they accelerate the uptake of $\mathrm{K}^{+}$ and the discharge of $\mathrm{Na}^{+}$to maintain the balance of these ions. A relatively high $\mathrm{K}^{+} / \mathrm{Na}^{+}$ratio can reduce salt-mediated damage to cell metabolism (Zhu, 2003). These pathways are $\mathrm{Ca}^{2+}-$ dependent (Leung and Giraudat, 1998; Sanders et al., 1999; Schroeder et al., 2001). It is crucial to identify and characterize the genes and their encoded proteins involved in this process to reveal the mechanisms of plant salt tolerance. Studies have shown that antiporter proteins encoded by the $N H X$ gene family, together with SOS1, SOS2, and SOS3, play a vital role in maintaining the balance between $\mathrm{Na}^{+}$and $\mathrm{K}^{+}$(Hasegawa et al., 2000; Quesada et al., 2000). Some studies have also shown that the $V D A C$ gene family is up-regulated and expressed under salt stress in rice (Bitar et al., 2003) and pearl millet (Desai et al., 2006). Wen et al. (2013) found that $\mathrm{Ca}^{2+}$ can rectify the imbalance of the salt stress response caused by Arabidopsis thaliana VDAC2 (AtVDAC2) disorders, restoring the reduced seed germination under salt stress to normal levels. However, knowledge of the molecular mechanism of VDAC gene regulation of salt stress response in plants is still poor (Godbole et al., 2003).

It is well known that plants can accumulate salicylic acid (SA) in their cells and then produce multiple response-associated molecules to increase tolerance to pathogenic microorganisms and resistance to infection (Alvarez, 2000). Under exogenous SA treatment, pathogenesis-related proteins accumulate quickly, which increases the resistance to pathogenic microorganisms. Among the response molecules, the pathogenesis-related gene $1(P R I)$ is the most important, as it is involved in many defense responses and is considered as the systemic acquired resistance marker gene (Penninckx et al., 1996; Dangl, 1998). Although some reports have suggested that $V D A C$ genes may be involved in the defense response of cells and cell 
death in plants (Desai et al., 2006; Kusano et al., 2009), there is little experimental evidence to corroborate whether $V D A C$ genes are actually involved in these processes. In addition, the mechanism of $V D A C$ gene participation in plant defense is still unclear.

In this study, we investigated the germination rate of transgenic plants under SA stress, and detected expression levels of signal related-genes to clarify the role of AtVDAC2 in the salt stress response and defense process. Our study revealed the mechanism of AtVDAC2 regulation on salt stress signal transduction and the SA signaling pathway, thereby laying the foundation to elucidate the plant stress response process.

\section{MATERIAL AND METHODS}

\section{Plant materials}

These sense and anti-sense AtVDAC2 transgenic Arabidopsis plants were obtained using the methods as described by Yan et al. (2009). Plants were grown on germination medium agar plates to seedlings, and then transferred to culture pots containing moist soil comprised of $50 \%$ nutrient soil $/ 50 \%$ vermiculite (1:1), together with $1 / 2 \mathrm{MS}$ (Murashige and Skoog basal medium). All plants were grown in a growth chamber with a 16 -h photoperiod at $23 \pm 2^{\circ} \mathrm{C}$ for 4 weeks.

Young leaves of 30-day-old transgenic and wild-type Arabidopsis plants were sampled to isolate total RNA with a plant RNA isolation kit (Tiangen Biotech, Beijing, China). The RNA was further reverse-transcribed to cDNA using a PrimeScript reverse transcription polymerase chain reaction (RT-PCR) Kit (TaKaRa Bio., Otsu, Shiga, Japan), and quantitative real-time PCR (qPCR) was performed using the SYBR Premix ExTaq ${ }^{\mathrm{TM}}$ II kit (TaKaRa) on a Bio-Rad IQ5 System (Bio-Rad Laboratories, Berkeley, CA, USA). The expression levels of AtVDAC2 in transgenic plants were detected with qRT-PCR using the primers AtVDAC2-Q, with $\beta$-actin as a reference. Data were collected with Icycler (Bio-Rad, USA). Each reaction was repeated three times. The comparable $\mathrm{Ct}$ (threshold) values were obtained to calculate the standard deviations. After the quantitative PCR, the melt curve was analyzed, so that the authenticity of the amplified product was verified. All raw data were analyzed by the Bio-Rad iQ5 software.

\section{Seed germination rate of $A t V D A C 2$ transgenic lines under SA treatment}

The seeds of AtVDAC2 transgenic Arabidopsis (V+2, V+4, V-2, and V-8) and the wild type (RLD) were first vernalized at $4{ }^{\circ} \mathrm{C}$ for 3 days, then seeds were surface sterilized in $75 \%$ ethanol for $45 \mathrm{~s}$, then treated with $0.1 \% \mathrm{HgCl}_{2}$ for $8 \mathrm{~min}$ and washed three times in sterile distilled water. At last, seeds were sown on the MS medium containing $3 \%$ sucrose and $0.8 \%$ agar, pH 5.9. For SA treatment, the sterilized seeds were sown on water-soaked (control) and 5 mM SA-soaked paper. Continuous observation and recording of germination were performed during a 10-day period. All germination rates and the variances were analyzed and calculated by Microsoft Excel 2003 (Microsoft, USA).

\section{Leaf stomata movement in transgenic Arabidopsis under $\mathrm{NaCl}$ treatment}

The 4-6-week-old leaves of the transgenic Arabidopsis were sampled and washed with distilled water, and then incubated in MES-KCl buffer $(10 \mathrm{mM}$ MES-KOH, $50 \mathrm{mM} \mathrm{KCl}$, $\left.0.05 \mathrm{mM} \mathrm{CaCl}_{2}, \mathrm{pH} 6.15\right)$. All plant samples were grown in a growth chamber with a light 
intensity of $0.1 \mathrm{mmol} \cdot \mathrm{m}^{-2} \cdot \mathrm{s}^{-1}$ at $23^{\circ} \mathrm{C}$ for 2 weeks to make the stomata open completely. The cultured leaves were picked out from the buffer and placed into fresh buffer containing 100 $\mathrm{mM} \mathrm{NaCl}$. After $30 \mathrm{~min}$, the samples were fixed on transparent plastic slides and the stomatal openings were examined under a microscope. The size of the stomatal opening was imaged under 10 x 40 optical microscopy and measured with the PhotoshopCS software. Each treatment was repeated three times, and then three to five images were randomly selected for measurement. The long and short axes of 30 to 40 stomata were recorded, and the ratio of long and short axes was calculated as the value of the stomatal opening. Finally, the relative stomatal openings of the transgenic plants were calculated using the wild type as the control. Each treatment was repeated four to six times. All stomatal closure and the variances were analyzed and calculated by Microsoft Excel 2003.

\section{Analysis of the expression level of salt stress- and SA-associated genes in transgenic Arabidopsis}

To test the regulatory function of VDAC2, we analyzed the expression levels of salt stress-associated genes (SOS1 and SOS2) in transgenic Arabidopsis using qPCR with genespecific primers (SOSl-Q and SOS2-Q) (Table 1). The Arabidopsis actin gene was selected as the reference. Total RNA isolation and qRT-PCR were performed using the methods described above. In addition, the expression level of the SA-associated gene $P R I$ was also analyzed with the primers PR1-Q (Table 1).

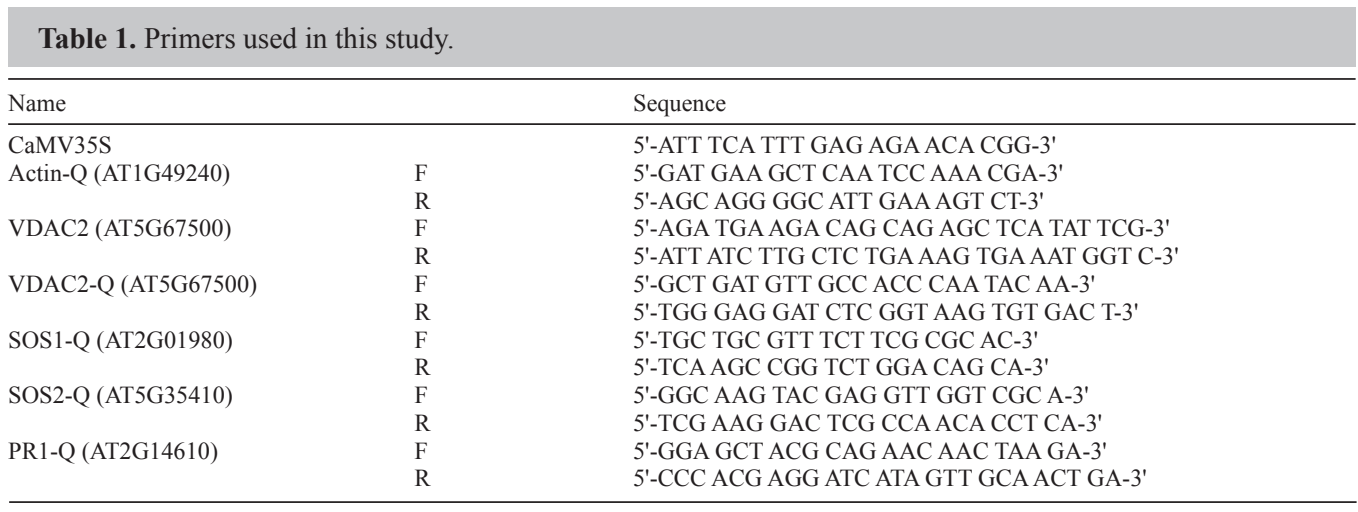

$\mathrm{F}=$ forward primer; $\mathrm{R}=$ reverse primer.

\section{RESULTS}

\section{AtVDAC2 expression level in transgenic Arabidopsis}

The expression level of the AtVDAC2 gene in the transgenic lines was detected with qPCR. Results showed that in sense lines, the expression levels of $A t V D A C 2$ were significantly increased $(\mathrm{P}<0.05)$, over 100 times those of the control, while in the antisense lines, the expression level of $A t V D A C 2$ dropped significantly to approximately $70 \%$ of control levels (Figure 1). This result indicated that we successfully obtained the AtVDAC2 transgenic Arabidopsis strain. 


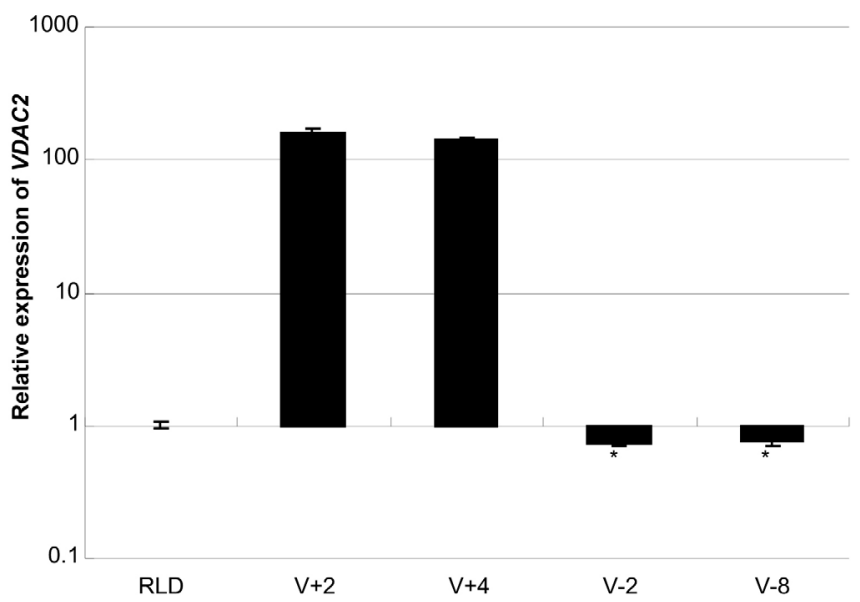

Figure 1. Relative expression level of $A t V D A C 2$ in transgenic lines measured by qRT-PCR. RLD: wild-type plants; $\mathrm{V}+2$ and $\mathrm{V}+4$ : overexpression lines; $V-2$ and $V-8$ : antisense lines. qRT-PCR: quantitative real-time reverse transcription polymerase chain reaction. The standard deviations were calculate by the Bio-Rad iQ5 software. ${ }^{* P}$ $<0.05$.

\section{Seed germination of AtVDAC2 transgenic Arabidopsis under SA treatment}

As shown in Figure 2, under normal conditions, more than $90 \%$ of seeds germinated in 3 days, and then all germinated on the 10th day. There was no difference between sense, antisense, and wild-type lines. Under $5 \mathrm{mM}$ SA treatment, a significant difference was observed on the third day $(\mathrm{P}<0.05)$. The over-expression lines showed lower seed germination rates than those of the wild type, suggesting that they were SA-sensitive, while the antisense lines had a higher germination rate, suggesting that they were SA-insensitive. Over time, the differences became narrower, until there was no difference by the 10th day. We postulated that the $V D A C$ gene was involved in the initial signal transduction process of seed germination in Arabidopsis.

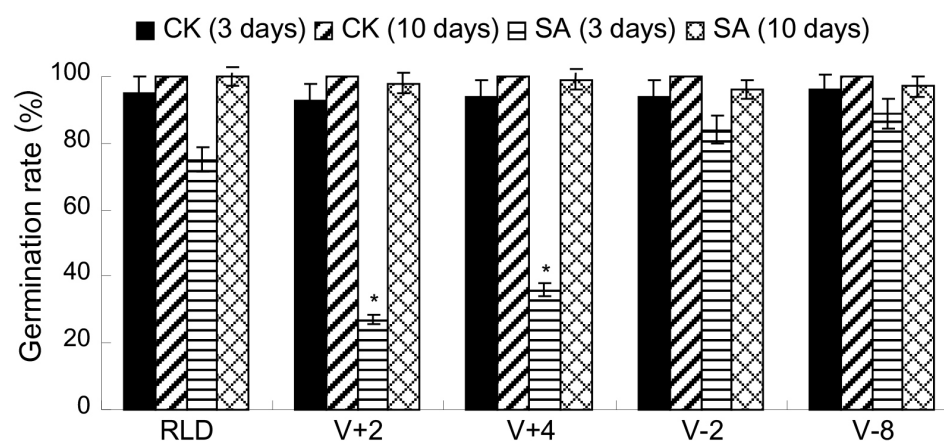

Figure 2. Seed germination rates of $A t V D A C 2$ transgenic lines under SA treatment. RLD: wild-type plants; $\mathrm{V}+2$ and $\mathrm{V}+4$ : overexpression lines; $V-2$ and $V-8$ : antisense lines; CK: control group treated with $\mathrm{H}_{2} \mathrm{O}$; $\mathrm{SA}$ : treatment group treated with $5 \mathrm{mM}$ salicylic acid (SA). Statistics were run on the third and tenth day of germination $(\mathrm{N}>$ 80). $* \mathrm{P}<0.05$. 


\section{Stomatal aperture of $A t V D A C 2$ transgenic plants under $\mathrm{NaCl}$ treatment}

We also investigated the stomatal aperture of the transgenic lines under salt treatment using the method described in the Material and Methods section (Figure 3). We found that $\mathrm{NaCl}$ salt stress affected stomatal movement of both wild-type and transgenic Arabidopsis, causing closure of the stomata. The over-expression lines showed sensitivity to $\mathrm{NaCl}$, and stomatal closure was obvious, at a size of about $80 \%$ of the RLD line. The antisense lines, which were not sensitive to $\mathrm{NaCl}$, showed no obvious change in the stomatal aperture, with a size of approximately $110 \%$ of the RLD line. It is well known that stomatal movement is influenced by moisture content in the environment, as water flows from high to low potential. Our results showed that the water potential became low when the solution contained $\mathrm{NaCl}$. Stomata can respond to the signal of water deficit, which sensitizes the stomata to water and causes them to close. Overall, the results indicated that AtVDAC2 is involved in the signal transduction process of the stomata response to $\mathrm{NaCl}$ in Arabidopsis.

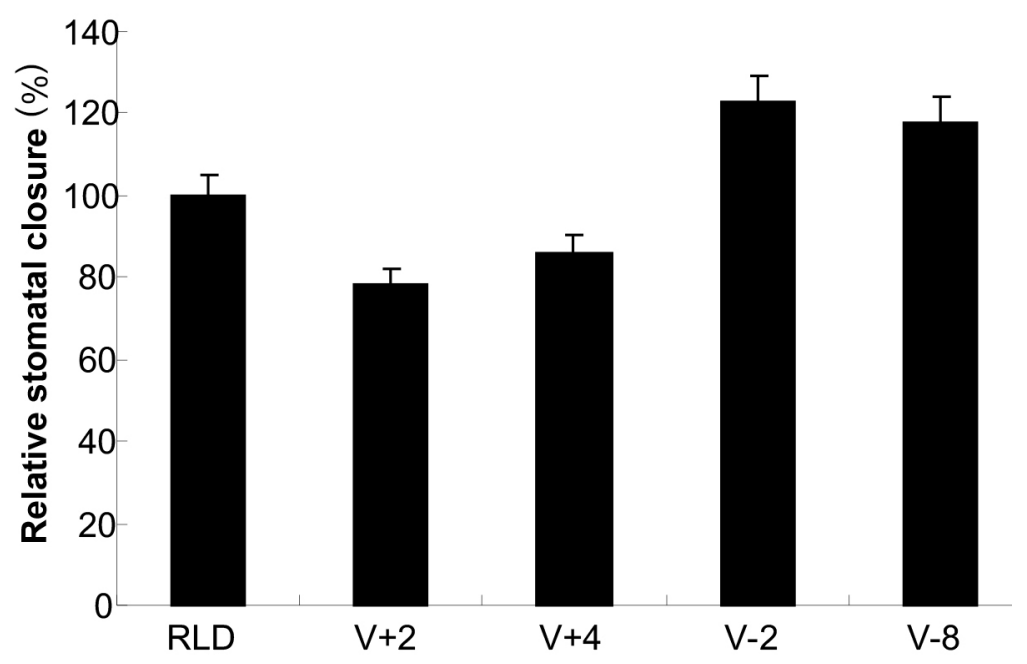

Figure 3. Stomatal aperture of $A t V D A C 2$ transgenic lines under $\mathrm{NaCl}$ treatment. RLD: wild-type plants; $\mathrm{V}+2$ and $\mathrm{V}+4$ : over-expression lines; $V-2$ and $V-8$ : antisense lines. The variances were calculate by Microsoft Excel 2003.

\section{Expression of salt- and SA-associated genes in AtVDAC2 transgenic Arabidopsis}

The SOS pathway is important for the plant response to salt stress. Our study found that AtVDAC2 played some role in the salt stress response, although the regulation mechanism was not clear. To understand the regulatory function of AtVDAC2, we detected the expression levels of the SOS1 and SOS2 genes, which are the main regulators in the SOS pathway (Figure 4A, B). Results showed that in the over-expression lines, the SOS1 and SOS2 genes were strongly expressed. In the $\mathrm{V}+4$ transgenic line, the expression level of these two genes was higher (200 and 70 times wild type), and in the V+2 line, expression was 20 and 10 times higher, respectively. In the antisense lines, the expression levels of SOS1 and SOS2 were very low, only $20 \%$ of the wild type. According to the above results, we postulated that AtVDAC2 was involved in the salt stress response in Arabidopsis through regulation of downstream 
signal molecules such as SOS1 and SOS2. Over-expression of AtVDAC2 might hasten signal transmission and indicate growth sensitivity under salt stress. Furthermore, the inhibited expression of AtVDAC2 might block signal transduction and prevent proper transmission, causing the salt stress signal to be ignored and thereby slow growth.

We also investigated the expression level of pathogenesis-related protein PR1 in $A t V D A C 2$ transgenic lines to reveal the role of AtVDAC2 in the SA response (Figure 4C). Results showed that the expression level of $P R 1$ increased by 15 to 20 times in transgenic lines compared to the wild type, and the expression of $P R 1$ was very weak in antisense lines. This suggested that VDAC affected expression of the PR1 gene. This study demonstrated that AtVDAC2 is involved in the signal transduction of SA response to produce the PR1 protein in Arabidopsis.
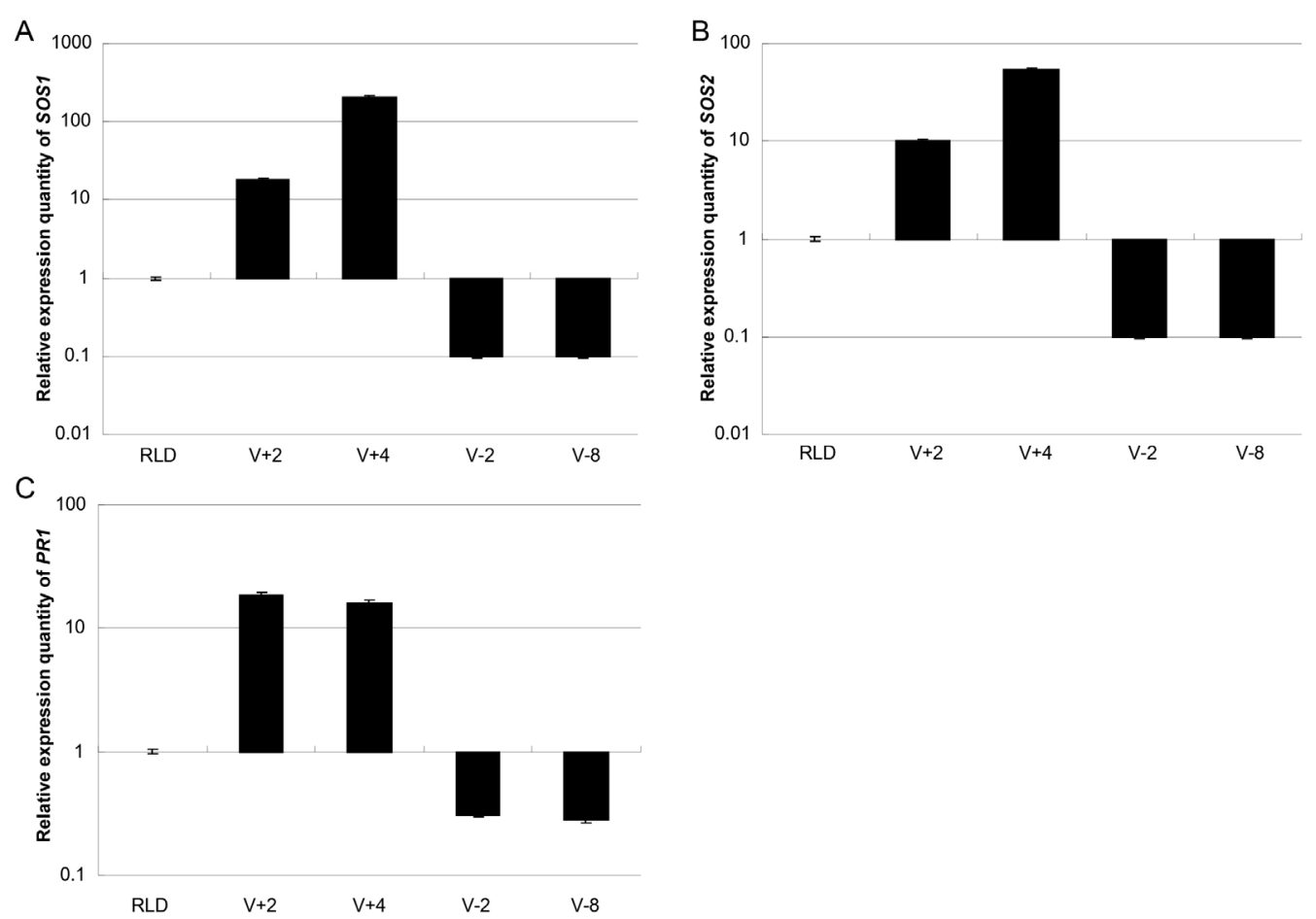

Figure 4. Relative expression levels of $S O S 1, S O S 2$, and $P R 1$ measured by real-time PCR. RLD: wild-type plants; $\mathrm{V}+2$ and V+4: over-expression lines; $V-2$ and $V$-8: antisense lines. The expressing quantity of AtVDAC2 and SOS3 was analyzed by $\triangle \mathrm{CT}$ method, $\triangle \mathrm{CT}$ was gotten by the CT of SOS1, SOS2, and PR1 to reduce the CT of $\beta$-actin, then the relative expressing values were accounted for by $\Delta \mathrm{CT}$. The standard deviations were calculate by the BioRad iQ5 software.

\section{DISCUSSION}

VDAC is the major transport protein in the outer membrane of mitochondria, which plays a crucial role in metabolite transport and in various signal transduction processes. Desai et al. (2006) reported that the expression of VDAC increased significantly under SA treatment within $1 \mathrm{~h}$. Lee et al. (2009) found that when Arabidopsis was infected by pathogens, VDAC expression increased quickly, and was accompanied by an increased expression of the $P R 1$ gene. 
In this study, we found that the overexpressed AtVDAC transgenic Arabidopsis lines were sensitive to SA, while the antisense lines were insensitive to SA. Furthermore, the expression levels of the $P R 1$ gene showed significant differences between the sense and antisense transgenic lines. Previous studies have concluded that plants can produce SA when infected by pathogenic microbials, resulting in strong expression of the $V D A C$ gene. This allows permeability transition pore to participate in the defense response in plants, such as through PR1 protein expression and $\mathrm{H}_{2} \mathrm{O}_{2}$ content increase. VDAC plays an important role in the regulation of mitochondrial metabolism. This study provides direct evidence for its additional function in plant disease defense. Wen et al. (2013) found that the over-expression of AtVDAC2 could increase the sensitivity to $\mathrm{NaCl}$ and regulated $\mathrm{SOS} 3$ gene expression which is involved in salt stress signaling pathway. These results indicate that AtVDAC2 is an important regulator in the pathway of Arabidopsis response to $\mathrm{NaCl}$ stress.

$\mathrm{Ca}^{2+}$ is an important second messenger in cells, and is involved in a wide range of cellular signal transduction. Studies have shown that salt ions can inhibit $\mathrm{Ca}^{2+}$ uptake and utilization, causing a Ca ${ }^{2+}$ deficit in plants under salt stress (Epstein and Rains, 1987). Salt stress damages plants mainly through destruction of the integrity and delivery process of the calcium signaling system on the plasma membrane (Cramer, 1985). Thus, providing the proper amount of exogenous calcium under salt stress has two effects. First, it can alleviate nutrition deficiency caused by calcium shortages. Second, calcium ions help to maintain normal operation and transmission of the plasma membrane, resulting in intracellular ion balance. Under salt stress, extracellular $\mathrm{Ca}^{2+}$ can reduce the absorption of $\mathrm{Na}^{+}$by the cell wall and plasma membrane, maintain the integrity and function of the plant cell membrane system, and increase the activity of $\mathrm{K}^{+}$channels on the plasma membrane. It can also decrease the $\mathrm{Na}^{+} / \mathrm{K}^{+}$ratio to alleviate the damage of salt stress.

This study elucidated the vital role of VDAC that involved in the salt stress and SA response process. This study opens the door for further research to reveal its molecular mechanism.

\section{ACKNOWLEDGMENTS}

Research supported by the National Natural Science Foundation of China (\#31171586 to Y. Yang; \#31271758 to J.M. Wang) and the National “973” Project (\#2013CB733903).

\section{REFERENCES}

Alvarez ME (2000). Salicylic acid in the machinery of hypersensitive cell death and disease resistance. Plant Mol. Biol. 44: 429-442.

Bitar F, Roosens N, Smeyers M, Vauterin M, et al. (2003). Sequence analysis, transcriptional and posttranscriptional regulation of the rice VDAC family. Biochem. Biophys. Acta 1625: 43-51.

Bonneau L, Ge Y, Drury GE and Gallois P (2008). What happened to plant caspases? J. Exp. Bot. 59: 491-499.

Cramer GR (1985). Displacement of $\mathrm{Ca}^{2+}$ and $\mathrm{Na}^{+}$form the plasmalemma of root cells. Plant Physiol. 79: 207-211.

Dangl J (1998). Innate immunity: plants just say NO to pathogens. Nature 394: 525-527.

Desai MK, Mishra RN, Verma D, Nair S, et al. (2006). Structural and functional analysis of a salt stress inducible gene encoding voltage dependent anion channel (VDAC) from pearl millet (Pennisetum glaucum). Plant Physiol. Biochem. 44: 483-493.

Douce R (1985). Mitochondria in Higher Plants: Structure, Function, and Biogenesis. 1st edn. Academic Press, Orlando. Douce R and Neuburger M (1989). The uniqueness of plant mitochondria. Annu. Rev. Plant Physiol. Plant Mol. Biol. 40: 371-414.

Dudkina NV, Heinemeyer J, Sunderhaus S and Boekema EJ (2006). Respiratory chain supercomplexes in the plant mitochondrial membrane. Trends Plant Sci. 11: 232-240. 
Epstein P and Rains DW (1987). Advance salt tolerance. Plant Soil 99: 17-29.

Godbole A, Varghese J, Sarin A and Mathew MK (2003). VDAC is a conserved element of death pathways in plant and animal systems. Biochem. Biophys. Acta 1642: 87-96.

Hasegawa PM, Bressan RA, Zhu JK and Bohnert HJ (2000). Plant cellular and molecular responses to high salinity. Annu. Rev. Plant Physiol. Plant Mol. Biol. 51: 463-499.

Hofius D, Tsitsigiannis DI, Jones JD and Mundy J (2007). Inducible cell death in plant immunity. Semin. Cancer Biol. 17: 166-187.

Kroemer G, Galluzzi L and Brenner C (2007). Mitochondrial membrane permeabilization in cell death. Physiol. Rev. 87: 99-163.

Kusano T, Tateda C, Berberich T and Takahashi Y (2009). Voltage-dependent anion channels: their roles in plant defense and cell death. Plant Cell Rep. 28: 1301-1308.

Lee SM, Hoang MH, Han HJ, Kim HS, et al. (2009). Pathogen inducible voltage-dependent anion channel (AtVDAC) isoforms are localized to mitochondria membrane in Arabidopsis. Mol. Cells 27: 321-327.

Leung J and Giraudat J (1998). Abscisic acid signal transduction. Annu. Rev. Plant Physiol. Plant Mol. Biol. 49: 199-222.

Love AJ, Milner JJ and Sadanandom A (2008). Timing is everything: regulatory overlap in plant cell death. Trends Plant Sci. 13: 589-595.

Mackenzie S and McIntosh L (1999). Higher plant mitochondria. Plant Cell 11: 571-586.

Moller IM (2001). Plant mitochondria and oxidative stress: electron transport, NADPH turnover, and metabolism of reactive oxygen species. Annu. Rev. Plant Physiol. Plant Mol. Biol. 52: 561-591.

Pennell RI and Lamb C (1997). Programmed cell death in plants. Plant Cell 9: 1157-1168.

Penninckx IA, Eggermont K, Terras FR, Thomma BP, et al. (1996). Pathogen-induced systemic activation of a plant defensin gene in Arabidopsis follows a salicylic acid-independent pathway. Plant Cell 8: 2309-2323.

Quesada V, Ponce MR and Micol JL (2000). Genetic analysis of salt-tolerant mutants in Arabidopsis thaliana. Genetics 154: 421-436.

Reape TJ and McCabe PF (2008). Apoptotic-like programmed cell death in plants. New Phytol. 180: 13-26.

Sanders D, Brownlee C and Harper JF (1999). Communicating with calcium. Plant Cell 11: 691-706.

Schroeder JI, Allen GJ, Hugouvieux V, Kwak JM, et al. (2001). Guard cell signal transduction. Annu. Rev. Plant Physiol. Plant Mol. Biol. 52: 627-658.

Wen GQ, Cai L, Liu Z, Li DK, et al. (2013). Arabidopsis thaliana VDAC2 involvement in salt stress response pathway. African J. Biotechnol. 10: 11588-11593.

Yan J, He H, Tong S, Zhang WR, et al. (2009). Voltage-dependent anion channel 2 of Arabidopsis thaliana (AtVDAC2) is involved in ABA-mediated early seedling development. Int. J. Mol. Sci. 10: 2476-2486.

Zhu JK (2003). Regulation of ion homeostasis under salt stress. Curr. Opin. Plant Biol. 6: 441-445. 